\title{
Genotyping of the G1138A mutation of the FGFR3 gene in patients with achondroplasia using high-resolution melting analysis
}

\author{
Chia-Cheng Hung ${ }^{\text {a, }}{ }^{\text {, Chien-Nan Lee }}{ }^{\text {b, }}$, Chien-Hui Chang ${ }^{\text {c }}$, Yuh-Jyh Jong ${ }^{\text {d,e }}$, \\ Chih-Ping Chen ${ }^{\text {f,g,h,i, }}$, Wu-Shiun Hsieh ${ }^{j}$, Yi-Ning Su ${ }^{k, 1, *}$, Win-Li Lin ${ }^{\text {a }}$

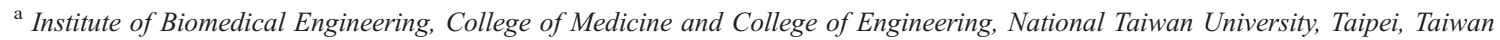 \\ ${ }^{\mathrm{b}}$ Department of Obstetrics and Gynecology, National Taiwan University Hospital, Taipei, Taiwan \\ ${ }^{\mathrm{c}}$ Institute of Molecular Medicine, College of Medicine, National Taiwan University, Taipei, Taiwan \\ ${ }^{\mathrm{d}}$ Department of Pediatrics, Kaohsiung Medical University Hospital, Kaohsiung, Taiwan \\ ${ }^{\mathrm{e}}$ Graduate Institute of Medicine, College of Medicine, Kaohsiung Medical University, Kaohsiung, Taiwan \\ ${ }^{\mathrm{f}}$ Department of Obstetrics and Gynecology, Mackay Memorial Hospital, Taipei, Taiwan \\ ${ }^{\mathrm{g}}$ Department of Medical Research, Mackay Memorial Hospital, Taipei, Taiwan \\ ${ }^{\mathrm{h}}$ Department of Biotechnology and Bioinformatics, Asia University, Taichung, Taiwan \\ ${ }^{i}$ College of Chinese Medicine, China Medical University, Taichung, Taiwan \\ ${ }^{\mathrm{j}}$ Department of Pediatrics, National Taiwan University Hospital, Taipei, Taiwan \\ ${ }^{\mathrm{k}}$ Graduate Institute of Clinical Medicine, College of Medicine, National Taiwan University, Taipei, Taiwan \\ ${ }^{1}$ Department of Medical Genetics, National Taiwan University Hospital, Taipei, Taiwan
}

Received 19 April 2007; received in revised form 21 July 2007; accepted 9 August 2007

Available online 6 September 2007

\begin{abstract}
Objectives: The fibroblast growth factor receptor 3 gene (FGFR3) plays a critical role in cartilage growth-plate differentiation and bony development. It has been shown that $97 \%$ of patients with achondroplasia have a $\mathrm{G}$ to A transition mutation at position 1138 (c.1138 $\mathrm{G}>\mathrm{A}$ ) of codon 380 of the FGFR3 gene.

Design and methods: Exon 8 of the FGFR3 gene was analyzed in 40 patients with achondroplasia, as well as in 50 control individuals for the presence of the $c .1138 \mathrm{G}>\mathrm{A}$ variant using melting curve analysis with a high-resolution melting instrument (HR-1).

Results: The high-resolution melting curve analysis successfully genotyped the c.1138G $>$ A mutation in exon 8 of the FGFR3 gene in all 40 patients with achondroplasia without the need of further assays. The technique had a sensitivity and specificity of $100 \%$.

Conclusion: High-resolution melting analysis is a simple, rapid, and sensitive one tube assay for genotyping the $F G F R 3$ gene. The technique is a low cost high-throughput FGFR3 screening assay.
\end{abstract}

(C) 2007 The Canadian Society of Clinical Chemists. Published by Elsevier Inc. All rights reserved.

Keywords: FGFR3 gene; Achondroplasia; Melting curve analysis

\section{Introduction}

Achondroplasia (OMIM\# 100800) is the most common form of short-limbed dwarfism in humans [1]. It is an autosomal

\footnotetext{
* Corresponding author. Graduate Institute of Clinical Medicine, College of Medicine, National Taiwan University, No. 7, Chung-Shan South Road, Taipei, Taiwan. Fax: +886223920470.

E-mail address: ynsu@ntu.edu.tw (Y.-N. Su).

${ }^{1}$ Chia-Cheng Hung and Chien-Nan Lee contributed equally to this study.
}

dominant disorder with full penetrance. The reported prevalence rate ranges from 1/15,000 to $1 / 70,000$ [2]. More than $90 \%$ of all cases are sporadic [3]. The characteristic phenotype of achondroplasia includes short stature, diminished muscle tone, lower part of the back, trident hand, limited elbow extension, and midface hypoplasia [4].

FGFRs represent a family of four tyrosine kinase receptors (FGFR1-4) that bind fibroblast growth factors. They are membrane proteins consisting of three extracellular glycosylated Iglike domains, a transmembrane domain, and a split cytoplasmic 
catalytic domain [5]. The fibroblast growth factor receptor 3 (FGFR3) gene is located on chromosome 4p16.3. Among the known mutations of this gene, a transition mutation c. $1138 \mathrm{G}>\mathrm{A}$ (Gly>Arg) at nucleotide 1138 in codon 380, in the transmembrane domain has been associated more than $97 \%$ of achondroplasia; only $1 \%$ of patients with achondroplasia have a $\mathrm{G}$ to $\mathrm{C}$ point mutation at the same site (c.1138G $>$ C) [6,7].

Most studies which have focused on SNPs of the FGFR3 gene associated with achondroplasia have used PCR-RFLP and realtime PCR to genotype thec.1138G $>$ A or c. $1138 \mathrm{G}>\mathrm{C}$ mutations. In general, the PCR-RFLP-based techniques are the most common approaches for the detection of single nucleotide polymorphism [8,9], but these are labor-intensive and cannot be used with high-throughput strategies. Real-time PCR and direct sequencing are rapid techniques [10]; however, they require relatively expensive reagent sets.

High-resolution melting curve analysis is a new approach for the detection of single nucleotide polymorphisms [11-13]. In recent years, several new instruments have been developed for the assay of DNA heterozygotes, including the Prism ${ }^{\circledR}$ 7900HT (Applied Biosystems), the LightCycler 1.2 and LightCycler 2.0 (Roche), the LightScanner ${ }^{\circledR}$ (Idaho Technology), and HR- ${ }^{\text {TM }}$ (Idaho Technology) [14]. Melting curve analysis with the high-resolution melting instrument (HR-1) is a simple, high-performance, reliable, high-resolution, time-saving, and low labor-intensive technique that has shown promise as a sensitive and specific tool for the detection of variations in DNA. Therefore, in this study, we analyze the sensitivity and specificity of genotyping FGFR3 gene variants by melting curve analysis with the HR-1 system. This approach allows for rapid and real-time detection of known single mutations. Moreover, denaturing high-performance liquid chromatography (DHPLC) is another promise tool for mutation screening [15-
17]. We also compare the sensitivity and specificity between the HR-1 system and DHPLC.

\section{Material and methods}

\section{Subjects and DNA extraction}

DNA samples were obtained from 40 patients with a diagnosis of achondroplasia with known genotypes and 50 unaffected individuals at the National Taiwan University Hospital. Genomic DNA was extracted from peripheral whole blood using the Chemagic DNA Blood Kit (Chemagen), according to the manufacturer's instructions.

\section{Primer design and PCR conditions}

PCR was conducted according to a protocol described previously [18]. PCR primers were designed to amplify G1138A mutations of the $F G F R 3$ gene for both melting curve analysis and DHPLC assay. Primer sequences were 5'-CCT CAA CGC CCA TGT CTT T-3' (sense) and 5'-AGG CAG CTC AGA ACC TGG TA-3' (anti-sense). The PCR products spanning exon 8 of the $F G F R 3$ gene generated a fragment of $249 \mathrm{bp}$. PCR was performed in a final volume of $25 \mu \mathrm{L}$ containing $100 \mathrm{ng}$ of genomic DNA, $100 \mu \mathrm{M}$ of each dNTP, $0.12 \mu \mathrm{M}$ each of primers, $0.5 \mathrm{U}$ of AmpliTaq Gold enzyme (PE Applied Biosystems), $2 \mathrm{mM} \mathrm{MgCl}_{2}$, and $2.5 \mu \mathrm{L}$ of $10 \times$ buffer II as provided by the manufacturer. For melting curve analysis, the PCR contains were modify to mix with $1 \times$ bovine serum albumin (BSA; Idaho Technology) and $1 \times \mathrm{LC}$ green dye (Idaho Technology). PCR was performed in an multiblock system (MBS) satellite thermal cycler (ThermoHybaid) with an initial denaturation step at $95{ }^{\circ} \mathrm{C}$ for $10 \mathrm{~min}$, followed by 35 cycles consisting of denaturation at $94^{\circ} \mathrm{C}$ for $30 \mathrm{~s}$, annealing at

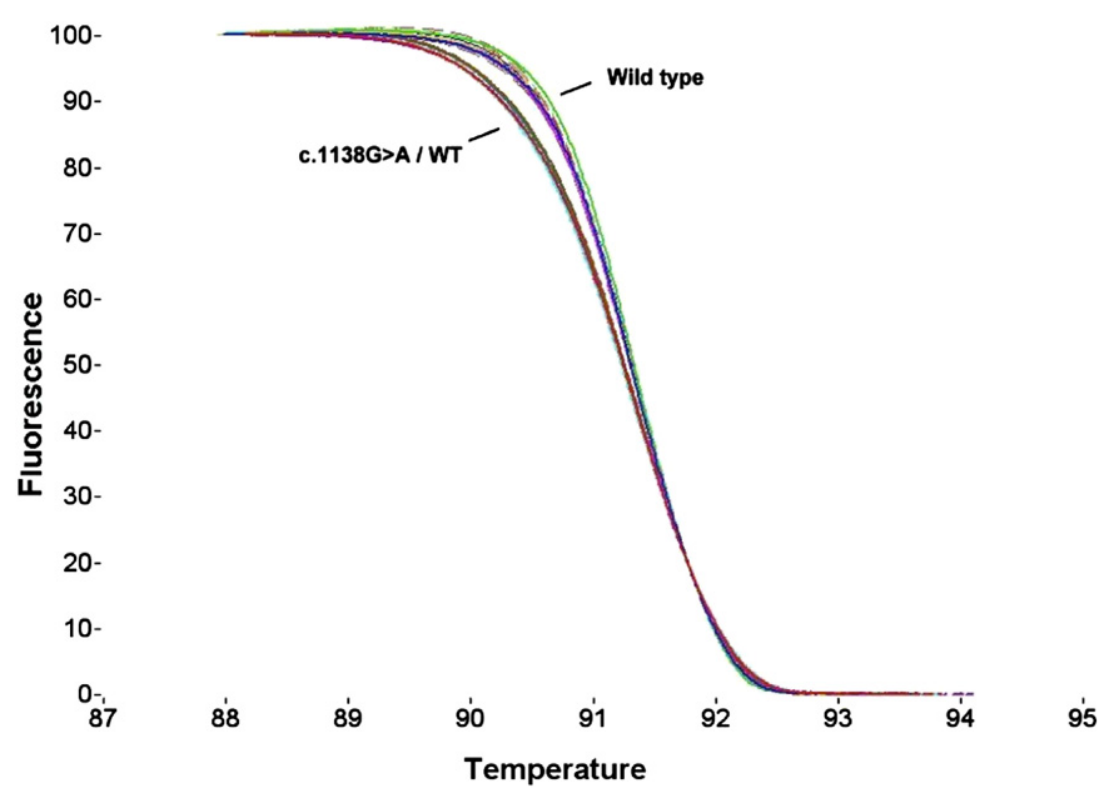

Fig. 1. Exon 8 of the FGFR3 gene was amplified from 40 patients and 50 unaffected individuals by high-resolution melting analysis. Sequencing confirmed that the wild type cluster and the c.1138G $>$ A clusters were different. 


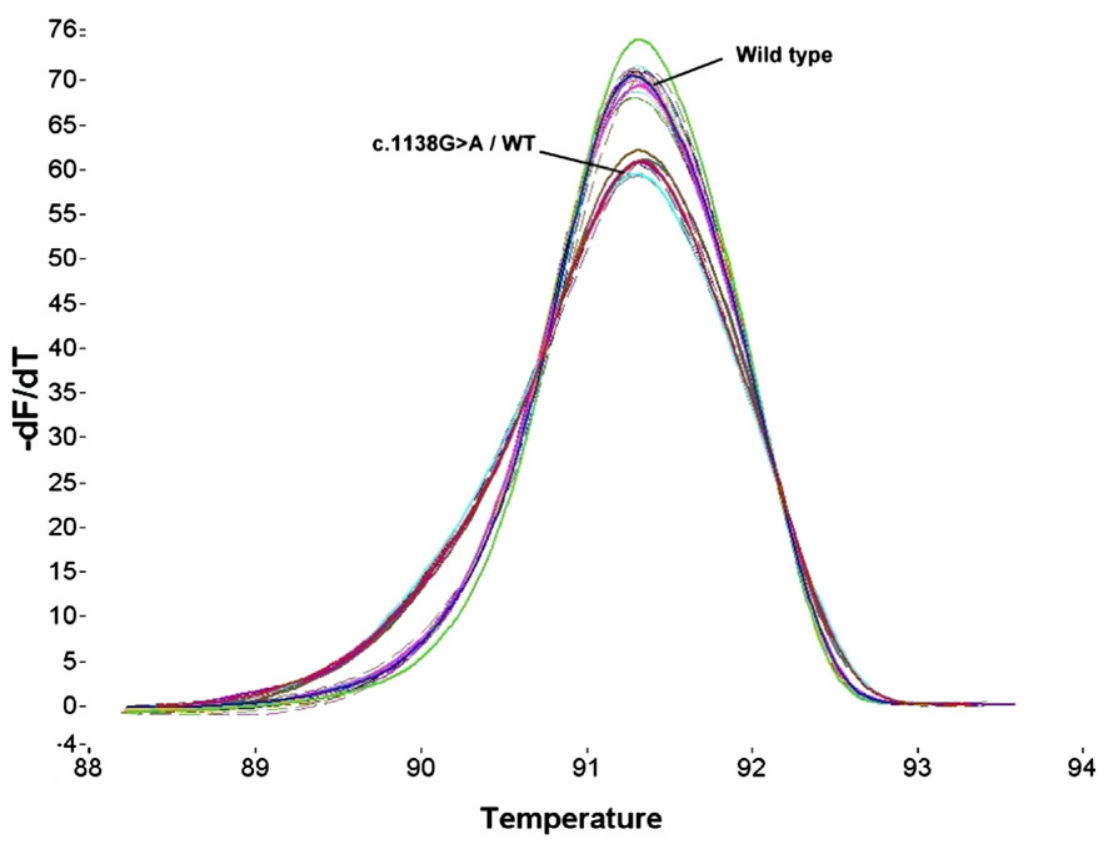

Fig. 2. After detection of the variant by high-resolution melting analysis, a derivative plot ( $-\mathrm{d} F / \mathrm{d} T$ vs. temperature) of Exon 8 of the $F G F R 3$ gene was constructed. Wild type clusters and c. $1138 \mathrm{G}>\mathrm{A}$ clusters melted at $90.5^{\circ} \mathrm{C}$.

$50{ }^{\circ} \mathrm{C}$ for $45 \mathrm{~s}$, elongation at $72{ }^{\circ} \mathrm{C}$ for $45 \mathrm{~s}$, and then a final extension step at $72{ }^{\circ} \mathrm{C}$ for $10 \mathrm{~min}$.

\section{DHPLC assay}

Heteroduplex analyses were performed according to the protocol of the manufacturer and previous studies [18,19]. PCR amplicons were performed under partially denaturing conditions in the Transgenomic Wave Nucleic Acid Fragment Analysis System (Transgenomic Inc.). PCR products were analyzed in a linear acetonitrile gradient with triethylammonium acetate as the mobile phase, using buffer A (0.1 M TEAA) and buffer B (0.1 M TEAA with $25 \%$ acetonitrile) (Transgenomic). Buffer concentrations at the start of the program were $53 \% \mathrm{~B}$, with a gradient over the $4.5-\mathrm{min}$ run time to $62 \% \mathrm{~B}$, and a flow rate of $0.9 \mathrm{~mL} / \mathrm{min}$. Run temperature was constant at $64.5{ }^{\circ} \mathrm{C}$, and eluted DNA fragments were detected at a wavelength of $260 \mathrm{~nm}$.

\section{Melting curve analysis}

When PCR amplification was complete, the PCR product was transferred to a LightCycler capillary. Melting curves were obtained by an initial holding step at $60{ }^{\circ} \mathrm{C}$ for $30 \mathrm{~s}$, followed by increasing the temperature slowly at a rate of $0.3^{\circ} \mathrm{C}$ per second to $96{ }^{\circ} \mathrm{C}$ with continuous fluorescence detection. The data were analyzed with custom software in which approximately 50 data points are acquired for every $1{ }^{\circ} \mathrm{C}$ as described previously. The melting curve data were normalized, temperature-shifted, and converted to melting peaks by plotting the negative derivative of the fluorescence with respect to temperature against temperature ( $-\mathrm{d} F / \mathrm{d} T$ vs. temperature).

\section{Results}

In this study, we extended the application of high-resolution melting curve analysis for easy and accurate genotyping of FGFR3 gene variants. A total of 40 patients with achondroplasia and 50 unaffected individuals were screened for the c.1138G $>A$ mutation using melting curve analysis (HR-1) and heteroduplex analysis (DHPLC). All sequence variants with c. $1138 \mathrm{G}>\mathrm{A}$ had been previously confirmed as exhibiting variations in the FGFR3 gene by direct sequencing analysis. The melting curves shown in Fig. 1 represent the two genotypes: wild type and heterozygote. None of the samples in our study had homozygous mutations.

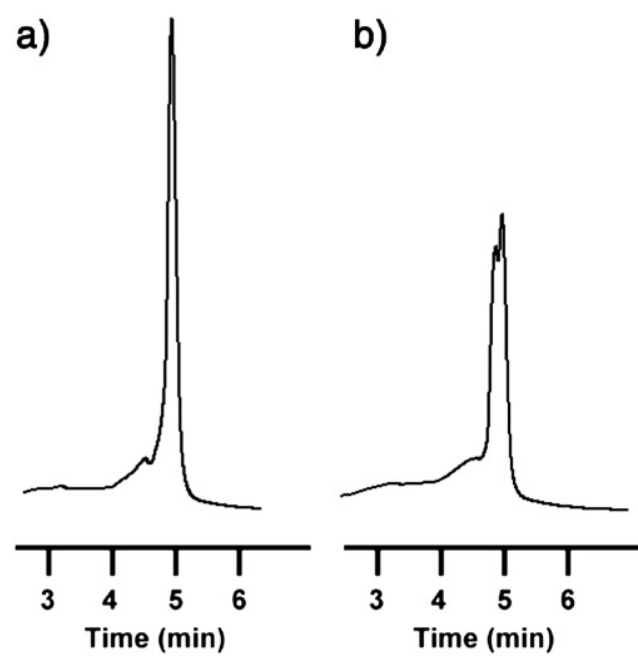

Fig. 3. DHPLC-based assay of the $F G F R 3$ gene (a) wild type and (b) heterozygous c. $1138 \mathrm{G}>$ A mutation. 
Fig. 2 shows the $-\mathrm{d} F / \mathrm{d} T$ vs. temperature that can distinguish the genotype. The wild type shows a single peak at $90.5^{\circ} \mathrm{C}$. However, the c.1138G $>$ A mismatch did not decrease the stability of the heteroduplex structure, and therefore resulted in the same melting temperature. The c.1138G $>$ A mismatch displays a single peak at $90.5^{\circ} \mathrm{C}$. Although the Tm of the mutations of the FGFR3 gene is close to the wild type on the derivative plot, the fluorescence difference plot demonstrates a clearer distinction of sequence variation. All 40 samples with c.1138G $>$ A mutation were identified successfully by melting curve analysis. In this study, melting curve analysis performed immediately after PCR and 1 week later resulted in reproducible chromatograms. Moreover, in order to identify and confirm the specific sequence variant of c.1138G $>$ A, we used DHPLC analysis to genotype the FGFR3 mutations. Fig. 3 shows the profile for each genotype obtained in DHPLC. A single peak was indicative of wild type homozygous individuals whereas two peaks denoted c. $1138 \mathrm{G}>\mathrm{A}$ heterozygous individuals. The results obtained from the melting curve and DHPLC analyses were further confirmed by direct sequencing; the sensitivity and specificity were both $100 \%$.

\section{Discussion}

The diagnosis of achondroplasia usually relies on the PCRRFLP method to screen for the presence of the c.1138G $>$ A mutation in the FGFR3 gene [8]. However, direct sequencing, realtime PCR [10], and matrix-assisted laser desorption/ionization time-of-flight mass spectrometry (MALDI-TOF MS) [20] have been reported to be more sensitive methods. Such molecular genetic testing is helpful in diagnosing genetic disorders.

Melting curve analysis is a novel, accurate, and simple technique for analyzing the human genome [21]. In this study, melting curve analysis using the HR-1 platform was $100 \%$ accurate in distinguishing FGFR3 gene variants from homozygous wild type samples. The major disadvantage of the protocol is the need to transfer the samples from PCR to capillary. The process carries a high risk of contamination. The protocol should be modified so that PCR is performed directly in an LC capillary, after which the sealed capillary can be transferred to the HR-1.

We compared the results of the melting analysis with those of DHPLC to screen for FGFR3 gene variants. The HR-1 system is a single tube instrument which efficiently analyzes one sample within a 2 min period. The automated DHPLC can analyze one sample in $10 \mathrm{~min}$ and 192 PCR products overnight. The cost to perform the melting curve analysis is approximately $\$ 1.50$ per sample, including capillary, PCR kit, and LCgreen dye kits. The cost of a single run of DHPLC is about $\$ 2.00$. Heterozygous mutations were detected by a change in shape in the melting curve analysis and DHPLC. In this study, all 40 samples with heterozygous mutations were identified correctly by melting curve analysis (100\% sensitivity), and 40 were identified accurately by DHPLC (100\% sensitivity). The specificities of both techniques were also $100 \%$. To test the accuracy of the two methods for genotyping, the individuals who analyzed the results obtained by the melting curve analysis and the DHPLC assay were blinded to the diagnoses of the patients. The patient and control groups received the experimental procedure at different times; neither group was made aware of when the experimental procedure had been given. We also analyzed each sample at least three times and all demonstrated reproducible results. Our results suggest that there are no differences in the diagnosis of the FGFR3 gene variants between melting curve analysis and DHPLC. Furthermore, the methods were automated to increase the speed, sensitivity, and reproducibility of the results, making it suitable for large-scale and high-throughput mutation screening.

Achondroplasia presents a clear phenotype, which is closely linked with a single mutation; therefore, diagnosing the disorder without lab tests poses little challenge to clinicians. Furthermore, the disorder is autosomal dominant with $100 \%$ penetrance. Therefore, the major genetic testing application will probably be antenatal screening, to identify affected fetuses, when one parent is affected, or, more commonly, in cases in which an achondroplastic couple has conceived; the homozygous condition is extremely severe, and is associated with early lethality. Here, we provide an alternative diagnostic tool. Once we establish the protocol, we can apply it to different single gene disorders.

The single nucleotide polymorphism (SNP) under investigation (codon 380) corresponds to the annotated SNP rs28931614 (gene ID: 2261). We amplified a $249 \mathrm{bp}$ fragment by PCR and performed a melting curve analysis of the amplified fragment. According to the databases, the amplified fragment according to base pairs 10,319 to 10,071 of the reference sequence (GenBank access no. AF487554) contains several other SNPs (e.g. rs11943863, rs17881656, rs28931615) affecting codons 383, 384, and 391. In addition, one of these SNPs has an overall high frequency of heterozygosity (1.1\%). Therefore, it is possible that the diagnostic strategy is potentially prone to error, particularly when DNA samples of subjects carrying these sequence variants/mutations are investigated. In addition, a novel heterozygous double mutation (c.1138_1139GG>AA) was recently described [22] that could potentially lead to misdiagnosis using the outlined strategy. However, melting curve analysis can detect sequence variants when compared with the wild type, which can be resolved by direct sequencing analysis in such situations. Moreover, according to previous studies $[12,23,24]$, the heteroduplex scanning technique is very sensitive when combined with the melting curve analysis. It can distinguish among different SNP genotypes.

One of the limitations of this study was the lack of a melting curve profile of the $\mathrm{c} .1138 \mathrm{G}>\mathrm{C}$ variant; unfortunately, we did not have any samples of that mutation. We assume that the c. $1138 \mathrm{G}>\mathrm{C}$ mutation could be distinguished from the wild type; however, the melting curve profile alone would probably not be able to differentiate between the c.1138G $>$ C and c.1138G $>$ A mutations because the melting effect would be very small, if not absent. Differentiation between the two would depend on the neighboring sequence context.

In summary, combined melting curve and heteroduplex analysis is a highly accurate, rapid, and efficient method for detecting the gene mutations.

\section{Acknowledgments}

The authors thank Dr. Fon-Jou Hsieh for his expert assistance. This work was supported by grants from the National Science 
Council of Taiwan (NSC 95-2314-B-002-280-MY3 and NSC 952314-B-002-034).

\section{References}

[1] Scott Jr CI. Achondroplastic and hypochondroplastic dwarfism. Clin Orthop Relat Res 1976:18-30.

[2] Bellus GA, Escallon CS, Ortiz de Luna R, et al. First-trimester prenatal diagnosis in couple at risk for homozygous achondroplasia. Lancet 1994;344:1511-2.

[3] Francomano CA. The genetic basis of dwarfism. N Engl J Med 1995;332:58-9.

[4] Vajo Z, Francomano CA, Wilkin DJ. The molecular and genetic basis of fibroblast growth factor receptor 3 disorders: the achondroplasia family of skeletal dysplasias, Muenke craniosynostosis, and Crouzon syndrome with acanthosis nigricans. Endocr Rev 2000;21:23-39.

[5] Green PJ, Walsh FS, Doherty P. Promiscuity of fibroblast growth factor receptors. Bioessays 1996;18:639-46.

[6] Bellus GA, Hefferon TW, Ortiz de Luna RI, et al. Achondroplasia is defined by recurrent G380R mutations of FGFR3. Am J Hum Genet 1995;56:368-73.

[7] Rousseau F, Bonaventure J, Legeai-Mallet L, et al. Mutations in the gene encoding fibroblast growth factor receptor-3 in achondroplasia. Nature 1994;371:252-4.

[8] Shiang R, Thompson LM, Zhu YZ, et al. Mutations in the transmembrane domain of FGFR3 cause the most common genetic form of dwarfism, achondroplasia. Cell 1994;78:335-42.

[9] Katsumata N, Mikami S, Nagashima-Miyokawa A, et al. Analysis of the FGFR3 gene in Japanese patients with achondroplasia and hypochondroplasia. Endocr J 2000;47 Suppl:S121-4.

[10] Schrijver I, Lay MJ, Zehnder JL. Rapid combined genotyping assay for four achondroplasia and hypochondroplasia mutations by real-time PCR with multiple detection probes. Genet Test 2004;8:185-9.

[11] Gundry CN, Vandersteen JG, Reed GH, Pryor RJ, Chen J, Wittwer CT. Amplicon melting analysis with labeled primers: a closed-tube method for differentiating homozygotes and heterozygotes. Clin Chem 2003;49:396-406.

[12] Reed GH, Wittwer CT. Sensitivity and specificity of single-nucleotide polymorphism scanning by high-resolution melting analysis. Clin Chem 2004;50:1748-54.
[13] Wittwer CT, Reed GH, Gundry CN, Vandersteen JG, Pryor RJ. Highresolution genotyping by amplicon melting analysis using LCGreen. Clin Chem 2003;49:853-60.

[14] Herrmann MG, Durtschi JD, Bromley LK, Wittwer CT, Voelkerding KV. Amplicon DNA melting analysis for mutation scanning and genotyping: cross-platform comparison of instruments and dyes. Clin Chem 2006;52: 494-503.

[15] Xiao W, Oefner PJ. Denaturing high-performance liquid chromatography: a review. Hum Mutat 2001;17:439-74.

[16] Hung CC, Chen CP, Lin SP, et al. Quantitative assay of deletion or duplication genotype by capillary electrophoresis system: application in Prader-Willi syndrome and Duchenne muscular dystrophy. Clin Chem 2006;52: 2203-10.

[17] Hung CC, Lee CN, Chen CP, et al. Quantification of relative gene dosage by single-base extension and high-performance liquid chromatography: application to the SMN1/SMN2 gene. Anal Chem 2005;77: 6960-8.

[18] Su YN, Lee CN, Chien SC, Hung CC, Chien YH, Chen CA. Rapid detection of FGFR3 gene mutation in achondroplasia by DHPLC systemcoupling heteroduplex and fluorescence-enhanced primer-extension analysis. J Hum Genet 2004;49:399-403.

[19] Su YN, Lee CN, Hung CC, et al. Rapid detection of beta-globin gene (HBB) mutations coupling heteroduplex and primer-extension analysis by DHPLC. Hum Mutat 2003;22:326-36.

[20] Li Y, Page-Christiaens GC, Gille JJ, Holzgreve W, Hahn S. Non-invasive prenatal detection of achondroplasia in size-fractionated cell-free DNA by MALDI-TOF MS assay. Prenat Diagn 2007;27:11-7.

[21] Montgomery J, Wittwer CT, Palais R, Zhou L. Simultaneous mutation scanning and genotyping by high-resolution DNA melting analysis. Nat Protoc 2007;2:59-66.

[22] Santos HG, Almeida M, Fernandes H, Wilkie A. Clinical hypochondroplasia in a family caused by a heterozygous double mutation in FGFR3 encoding GLY380LYS. Am J Med Genet A 2007;143:355-9.

[23] Vandersteen JG, Bayrak-Toydemir P, Palais RA, Wittwer CT. Identifying common genetic variants by high-resolution melting. Clin Chem 2007;53: 1191-8.

[24] Chou LS, Lyon E, Wittwer CT. A comparison of high-resolution melting analysis with denaturing high-performance liquid chromatography for mutation scanning: cystic fibrosis transmembrane conductance regulator gene as a model. Am J Clin Pathol 2005;124:330-8. 